\title{
Unimodal rays in the regular and generalized Pascal pyramids
}

\author{
Hacène Belbachir* \\ USTHB, Faculty of Mathematics \\ Po. Box 32 El Alia, Algiers, Algeria \\ hbelbachir@usthb.dz \\ László Szalay ${ }^{\dagger}$ \\ University of West Hungary, Institute of Mathematics and Statistics \\ H-9400 Sopron, Erzsébet utca 9., Hungary \\ laszalay@ktk. nyme.hu \\ Submitted: Jun 15, 2009; Accepted: Mar 25, 2011; Published: Mar 31, 2011 \\ Mathematics Subject Classification: 11B65, 11B83

\begin{abstract}
The present paper shows that any sequence of integers laying along a finite ray in any Pascal-pyramid is log-concave, consequently unimodal. Further, we describe an algorithm which provides the plateaus of the diagonal sequences locating on layer $n$ of the regular Pascal pyramid.
\end{abstract}

\section{Introduction}

Let $r \geq 2$ denote an integer, and consider the map $p: \mathbb{N}^{r} \rightarrow \mathbb{N}$,

$$
\left(x_{1}, \ldots, x_{r}\right) \mapsto\left(\begin{array}{c}
x_{1}+\cdots+x_{r} \\
x_{1}, \ldots, x_{r}
\end{array}\right)=\frac{\left(x_{1}+\cdots+x_{r}\right) !}{x_{1} ! \cdots x_{r} !}
$$

Clearly, the map $p$ provides the number of the ways of splitting a set of $x=x_{1}+\cdots+x_{r}$ distinguishable objects into pairwisely disjoint subsets $S_{i}$ with cardinality $x_{i}(i=1, \ldots, r)$, therefore

$$
\left(\begin{array}{c}
x_{1}+\cdots+x_{r} \\
x_{1}, \ldots, x_{r}
\end{array}\right)=\left(\begin{array}{c}
x_{1}+\cdots+x_{r} \\
x_{1}
\end{array}\right)\left(\begin{array}{c}
x_{2}+\cdots+x_{r} \\
x_{2}
\end{array}\right) \cdots\left(\begin{array}{c}
x_{r-1}+x_{r} \\
x_{r-1}
\end{array}\right)\left(\begin{array}{c}
x_{r} \\
x_{r}
\end{array}\right)
$$

${ }^{*}$ The research is supported by LAID3 Laboratory of USTHB University, and by Algerian-French bilateral CMEP-Tassili 09MDU765.

${ }^{\dagger}$ The research is supported by János Bolyai Scholarship of HAS, by Hungarian National Foundation for Scientific Research Grant No. T 61800 FT, and by Mexican-Hungarian bilateral TeT. 
holds with the usual binomial coefficients on the right hand side. When $r=2$, the map returns the regular binomial coefficients in the Pascal triangle. We obtain the regular Pascal pyramid if $r=3$. For the cases $r \geq 4$ we will refer as generalized Pascal pyramids.

The main purpose of this paper is to investigate the question of unimodality in Pascal pyramids. It has already been shown that the elements laying along any ray in the Pascal triangle form unimodal sequence (see [2]). Scrutinies were made to reveal the localization of certain rays' modes (for instance, see [5, 3, 1]). Here, in the first part we prove, similarly to Pascal triangle, that any ray crosses any Pascal pyramid is puncturing elements of an unimodal sequence. Later we concentrate only on the 3D case, where $r=3$, and consider the elements $\left(\begin{array}{c}x_{1}+x_{2}+x_{3} \\ x_{1}, x_{2}, x_{3}\end{array}\right)$ to specify certain modes when $n=x_{1}+x_{2}+x_{3}$ is fixed. More precisely, we describe the peaks and plateaus of the so called diagonal sequences on level $n$ in the pyramid. Figure 2 shows the first few levels of Pascal pyramid.

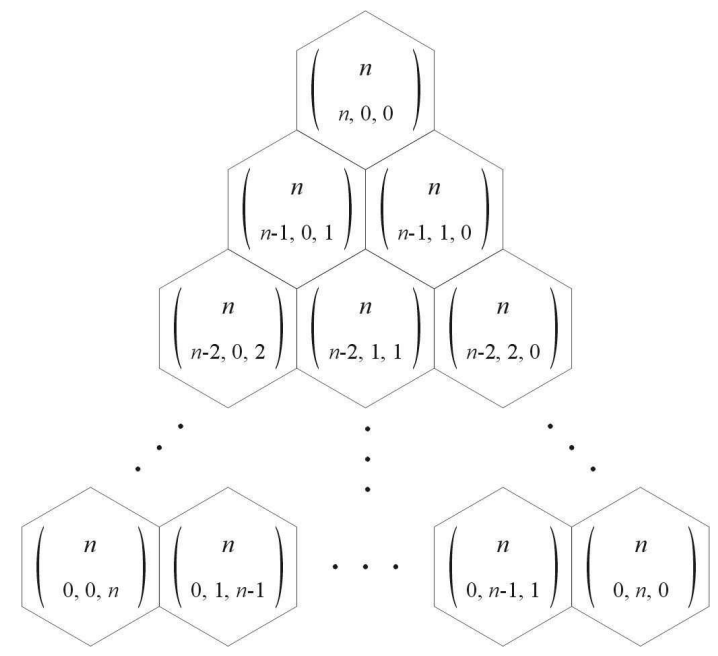

Figure 1: $n^{\text {th }}$ level of Pascal pyramid

Let $\omega$ denote a positive integer or the infinity. A real sequence $\left\{a_{k}\right\}_{k=0}^{\omega}$ is unimodal if there exist a non-negative integer $\lambda$ such that the subsequence $\left\{a_{k}\right\}_{k=0}^{\lambda}$ increases, while $\left\{a_{k}\right\}_{k=\lambda}^{\omega}$ decreases. When $\lambda=0$ then the sequence is monotone decreasing. Therefore it is also natural to consider a monotone increasing sequence as unimodal with $\lambda=\omega$, even if $\omega=\infty$.

If $a_{0} \leq a_{1} \leq \cdots \leq a_{m-1}<a_{m}=\cdots=a_{M}>a_{M+1} \geq a_{M+2} \geq \cdots$ then the integers $m, \ldots, M$ are called the modes of the sequence. In case of $m=M$, we talk about peak, otherwise the set of modes is called plateau.

A non-negative real sequence $\left\{a_{k}\right\}$ is logarithmically concave (log-concave or $L C$ for short) if

$$
a_{k}^{2} \geq a_{k-1} a_{k+1}
$$

holds for any $k \geq 1$.

By Theorem 1, log-concavity provides an opportunity to show unimodality. In the proof of Theorem 5 we use it together with Theorem 2. 
Theorem 1 A log-concave sequence $\left\{a_{k}\right\}$ with no internal zeros is also unimodal (see, for instance, [4]).

Theorem 2 The sequence of binomial coefficients located along a ray is log-concave (see [2]).

Now we recall an other important result, due to Wang and Yeh [6], which we can compose a log-concave sequence from initial log-concave sequences by.

Theorem 3 If m sequences $\left\{U_{k}^{(1)}\right\}, \ldots,\left\{U_{k}^{(m)}\right\}$ are all log-concave, then so is the sequence

$$
U_{n}=\sum\left(\begin{array}{c}
n \\
k_{1}, \ldots, k_{m}
\end{array}\right) U_{k_{1}}^{(1)} \cdots U_{k_{m}}^{(m)}, \quad n \in \mathbb{N},
$$

where the sum is over all non-negative integers $k_{1}, \ldots, k_{m}$ such that $k_{1}+\cdots+k_{m}=n$.

In order to prepare the proof of Theorem 5, let introduce the notation of rays in generalized Pascal pyramids. As usual, a lattice ray in the domain $\mathcal{D}_{p}$ of the function $p$ is given by two grid points $\vec{x}=\left(x_{1}, \ldots, x_{r}\right) \in \mathcal{D}_{p}$ and $\vec{y}=\left(y_{1}, \ldots, y_{r}\right) \in \mathcal{D}_{p}$, thus we consider the sequence

$$
s_{\vec{x}, \vec{y}}(k)=\left\{\left(\begin{array}{c}
\sum_{i=1}^{r} x_{i}+k \sum_{i=1}^{r} \alpha_{i} \\
x_{1}+k \alpha_{1}, \ldots, x_{r}+k \alpha_{r}
\end{array}\right)\right\}_{k}
$$

with $k \in \mathbb{N}$, where $\alpha_{i}=y_{i}-x_{i},(i=1, \ldots, r)$.

We also need a combinatorial argument to extend the Vandermonde identity

$$
\left(\begin{array}{l}
n \\
\nu
\end{array}\right)=\sum_{i}\left(\begin{array}{l}
k \\
i
\end{array}\right)\left(\begin{array}{l}
n-k \\
\nu-i
\end{array}\right)
$$

linked to the Pascal triangle as follows.

Theorem 4 Given the non-negative integers $n \geq k, n_{1}, \ldots, n_{r}$ such that $n_{1}+\cdots+n_{r}=n$. Then

$$
\left(\begin{array}{c}
n \\
n_{1}, \ldots, n_{r}
\end{array}\right)=\sum_{k_{1}, \ldots, k_{r}}\left(\begin{array}{c}
k \\
k_{1}, \ldots, k_{r}
\end{array}\right)\left(\begin{array}{c}
n-k \\
n_{1}-k_{1}, \ldots, n_{r}-k_{r}
\end{array}\right)
$$

holds, where $k_{1}+\cdots+k_{r}=k$ is satisfied on all possible manners while summing.

Proof: Suppose that we have $n$ students, $k$ and $n-k$ girls and boys, respectively. Assume that they have $r$ levels of studies (for example, first year, second year, etc.). We would like to distribute $n$ students such that $n_{i}$ of them are in level $i$. To get the number of all choices, we should only sum over all different possibilities to choose $k_{i}$ girls and $n_{i}-k_{i}$ boys to each level $i$. 


\section{Results and proofs}

Using the notation above, the principal result of this work is

Theorem 5 The sequence

$$
s_{\vec{x}, \vec{y}}(k)=\left\{\left(\begin{array}{c}
\sum_{i=1}^{r} x_{i}+k \sum_{i=1}^{r} \alpha_{i} \\
x_{1}+k \alpha_{1}, \ldots, x_{r}+k \alpha_{r}
\end{array}\right)\right\}_{k}, \quad k=0,1,2, \ldots
$$

is unimodal.

Proof: An immediate consequence of Theorem 4 if one applies the decomposition in (1) to $s_{\vec{x}, \vec{y}}(k)$ of $(3)$ is

$$
\left(\begin{array}{c}
\sum_{i=1}^{r} x_{i}+k \sum_{i=1}^{r} \alpha_{i} \\
x_{1}+k \alpha_{1}, \ldots, x_{r}+k \alpha_{r}
\end{array}\right)=\sum_{\substack{k_{1}, \ldots, k_{r} \\
k_{1}+\cdots+k_{r}=k}}\left(\begin{array}{c}
k \\
k_{1}, \ldots, k_{r}
\end{array}\right) \cdot U_{k_{1}}^{(1)} \cdot U_{k_{2}}^{(2)} \cdots U_{k_{r}-1}^{(r-1)} \cdot U_{k_{r}}^{(r)}
$$

where

$$
\begin{aligned}
U_{k_{1}}^{(1)} & =\left(\begin{array}{c}
\sum_{i=1}^{r} x_{i}+k \sum_{i=1}^{r} \alpha_{i}-k \\
x_{1}+k \alpha_{1}-k_{1}
\end{array}\right) \\
U_{k_{2}}^{(2)} & =\left(\begin{array}{c}
\sum_{i=2}^{r} x_{i}+k \sum_{i=2}^{r} \alpha_{i}-\left(k-k_{1}\right) \\
x_{2}+k \alpha_{2}-k_{2}
\end{array}\right) \\
& \vdots \\
U_{k_{r-1}}^{(r-1)} & =\left(\begin{array}{c}
\sum_{i=r-1}^{r} x_{i}+k \sum_{i=r-1}^{r} \alpha_{i}-\left(k-\sum_{i=1}^{r-2} k_{i}\right) \\
x_{r-1}+k \alpha_{r-1}-k_{r-1}
\end{array}\right) \\
U_{k_{r}}^{(r)} & =\left(\begin{array}{c}
x_{r}+k \alpha_{r}-k_{r} \\
x_{r}+k \alpha_{r}-k_{r}
\end{array}\right)=1 .
\end{aligned}
$$

Each sequence $\left\{U_{k_{i}}^{(i)}\right\}$ consists of certain binomial coefficients laying on a ray, hence by Theorem 2, it is log-concave. Now, applying Theorem 3, it follows that the sequence $s_{\vec{x}, \vec{y}}(k)$ is log-concave. Thus, by Theorem 1 , the proof is complete.

Put $n=q r+\varrho, 0 \leq \varrho<r$, where $n$ and $r \leq n$ are fixed positive integers. We even remark, that the maximum value

$$
\max _{n_{1}+\cdots+n_{r}=n}\left(\begin{array}{c}
n \\
n_{1}, n_{2}, \ldots, n_{r}
\end{array}\right)=(\underbrace{q+1, \ldots, q+1}_{\varrho \text { times }}, \underbrace{q, \ldots, q}_{r-\varrho \text { times }}) .
$$

In the sequel, we consider the regular Pascal pyramid, where $r=3$. Let $n \in \mathbb{N}$ be fixed, and $n=k_{1}+k_{2}+k_{3}$. In this case we will determine a sort of ray's peak and 
plateau in the pyramid. Obviously, there are finitely many non-negative integer triples $\left(k_{1}, k_{2}, k_{3}\right)=\left(k_{1}, k_{2}, n-k_{1}-k_{2}\right)$ satisfying the condition above.

Now, put $k_{1}=n-t($ with $0 \leq t \leq n), k_{2}=k$, consequently we have $k_{3}=n-k_{1}-k_{2}=$ $t-k$, further

$$
\left(\begin{array}{c}
n \\
k_{1}, k_{2}, n-k_{1}-k_{2}
\end{array}\right)=\left(\begin{array}{c}
n \\
n-t, k, t-k
\end{array}\right)=\left(\begin{array}{c}
n \\
n-t
\end{array}\right)\left(\begin{array}{l}
t \\
k
\end{array}\right)=\left(\begin{array}{l}
n \\
t
\end{array}\right)\left(\begin{array}{l}
t \\
k
\end{array}\right)
$$

hold.

It can be interpreted as the $k^{t h}$ element of the $t^{\text {th }}$ row on the $n^{\text {th }}$ layer of the Pascal pyramid. Clearly, $0 \leq k \leq t \leq n$. Therefore if someone takes the first $n$ rows of the Pascal triangle, and for each non negative integer $t \leq n$, multiplies the $t^{t h}$ row in it by the binomial coefficient $\left(\begin{array}{l}n \\ t\end{array}\right)$ then the $n^{\text {th }}$ layer in the Pascal pyramid is obtained.

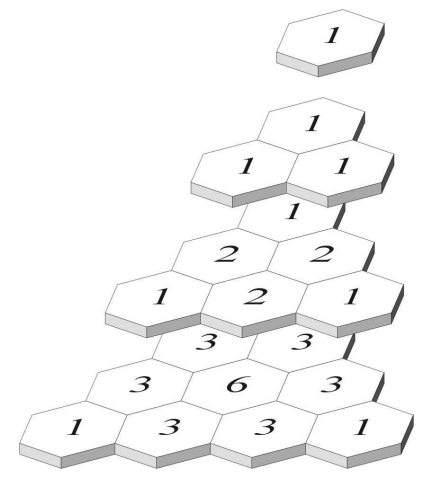

Figure 2: Pascal pyramid

It is well known that if $t$ is even, then the $t^{\text {th }}$ row of the Pascal triangle has a peak at $t / 2$, otherwise (when $t$ is odd) there is a plateau with the two elements $\lfloor t / 2\rfloor$ and $\lceil t / 2\rceil$. The situation is the same in the $n^{\text {th }}$ level of the Pascal pyramid, since each row here is a constant multiple of the corresponding row of the Pascal triangle (to see level $n$, look at Figure 1). The so called diagonal sequences have more interests. We refer to TANNY and ZUKER [5], who showed in the ordinary Pascal triangle that $\left(\begin{array}{c}n-k \\ k\end{array}\right)(k=0, \ldots,\lfloor n / 2\rfloor)$ is unimodal, and the authors describe the peaks and the plateaus with two elements, the elements in which the monotonicity changes. Analogously, for any $2 \leq t \leq n$, we investigate the diagonal sequence

$$
d_{t}(k)=\left(\begin{array}{c}
n \\
t-k
\end{array}\right)\left(\begin{array}{c}
t-k \\
k
\end{array}\right), \quad 0 \leq k \leq t
$$

of the $n^{\text {th }}$ level of the Pascal pyramid.

In the forthcoming part we deal with the peaks and plateaus of diagonal sequences $d_{t}(k)$ defined above. The first result classifies the possible cases, then we show how to determine the plateaus. 
Theorem 6 Given the positive integers $2 \leq t \leq n$, and put

$$
\kappa=\frac{(n+3 t)-\sqrt{(n+3 t)^{2}-12\left(t^{2}-n-1\right)}}{6} .
$$

Then $\kappa$ is a real number. If $t<\sqrt{n+1}$ then $\kappa<0$ and the sequence $d_{t}(k)$ is strictly monotone decreasing. If $\kappa$ is non-negative integer, then $d_{t}(k)$ has a plateau with the two elements $\kappa$ and $\kappa+1$. Finally, if $\kappa$ is a non-integer positive real number, then $d_{t}(k)$ possesses a peak at $\lceil\kappa\rceil$.

Proof: The condition $d_{t}(k) \leq d_{t}(k+1)$ is equivalent to

$$
0 \leq 3 k^{2}-(n+3 t) k+\left(t^{2}-n-1\right) .
$$

Since $2 \leq t \leq n$, the discriminant $D=(n+3 t)^{2}-12\left(t^{2}-n-1\right)$ of the equation

$$
0=3 k^{2}-(n+3 t) k+\left(t^{2}-n-1\right)
$$

is larger then $(4 t)^{2}-12\left(t^{2}-n-1\right)=4 t^{2}+12 n+12>0$. Thus (6) has two distinct real roots:

$$
\kappa_{ \pm}=\frac{(n+3 t) \pm \sqrt{D}}{6} .
$$

Applying again $t \leq n$, it is obvious, that for the larger one, say

$$
\kappa_{+}=\frac{(n+3 t)+\sqrt{(n+3 t)^{2}-12\left(t^{2}-n-1\right)}}{6} \geq \frac{(4 t)+\sqrt{4 t^{2}+12 t+12}}{6}>t
$$

hold, and we arrived at a contradiction since $\kappa \leq t$ should be satisfied (because $0 \leq k \leq t$ from $\left.d_{t}(k)\right)$. Put $\kappa=\kappa_{-}$. The reader readily can show that $\kappa<0$ holds if and only if $t<\sqrt{n+1}$. Thus the further parts of the theorem are obvious.

Now, we turn our attention to the question of modes with two elements. Plateau of the sequence $d_{t}(k)$ exists if and only if

$$
\kappa=\frac{(n+3 t)-\sqrt{D}}{6}
$$

is an integer satisfying $0 \leq \kappa \leq t / 2-1$. Hence $D$ necessarily must be a square, that is $D=$ $u^{2}$ holds for a suitable non-negative integer $u$. Note, that $D=(n+3 t+6)^{2}-12(t+1)(t+2)$, so we obtain

$$
(n+3 t+6-u)(n+3 t+6+u)=12(t+1)(t+2) .
$$

Put $a_{-}=n+3 t+6-u$ and $a_{+}=n+3 t+6+u$. Since both $a_{-}$and $a_{+}$are of the same parity and $a_{-} a_{+}$is even, $a_{-}$and $a_{+}$must both be even. Thus $a_{-} / 2$ and $a_{+} / 2$ are integers, one of them is divisible by 3 . Set $h_{1}, h_{2} \in \mathbb{N}$ such that $h_{1} h_{2}=3$ and $a_{-} / 2 h_{1} \in \mathbb{N}$, $a_{+} / 2 h_{2} \in \mathbb{N}$. Clearly,

$$
\frac{a_{-}}{2 h_{1}} \cdot \frac{a_{+}}{2 h_{2}}=(t+1)(t+2) .
$$


All the solutions of (8) can be represented as $t+1=s_{1} s_{2}, t+2=s_{3} s_{4}$ and $a_{-} / 2 h_{1}=s_{1} s_{3}$, $a_{+} / 2 h_{2}=s_{2} s_{4}$ for suitable positive integers $s_{i}, i=1, \ldots, 4$. It follows, that

$$
\begin{aligned}
u & =-h_{1} s_{1} s_{3}+h_{2} s_{2} s_{4}, \\
n & =h_{1} s_{1} s_{3}+h_{2} s_{2} s_{4}-3 s_{3} s_{4}, \\
t & =s_{3} s_{4}-2=s_{1} s_{2}-1,
\end{aligned}
$$

further

$$
\kappa=\frac{h_{1} s_{1} s_{3}}{3}-1
$$

Assume now, that $s_{1}$ and $s_{2}$ are given, and then determine all positive divisor pairs $\left(s_{3}, s_{4}\right)$ such that $s_{3} s_{4}=s_{1} s_{2}+1$. Then the parameters $s_{i}$ and $h_{1}, h_{2}$ generate $u, n, t$ and $\kappa$ as above. If the conditions $\sqrt{n+1} \leq t \leq n, 0 \leq \kappa \leq t / 2-1$ and $u \geq 0$ hold then a plateau is provided by

$$
\left(\begin{array}{c}
n \\
t-\kappa
\end{array}\right)\left(\begin{array}{c}
t-\kappa \\
\kappa
\end{array}\right)=\left(\begin{array}{c}
n \\
t-\kappa-1
\end{array}\right)\left(\begin{array}{c}
t-\kappa-1 \\
\kappa+1
\end{array}\right)
$$

In this manner, all plateaus can be obtained. Observe, that by (7), $3 \mid n$ implies $3 \mid u$, hence both $a_{-}$and $a_{+}$are also divisible by 3 . Therefore, to avoid the repetition in producing the plateaus, we must omit exactly one of the cases $\left(h_{1}, h_{2}\right)=(1,3)$ and $\left(h_{1}, h_{2}\right)=(3,1)$.

\section{Experimental observations on plateaus}

\begin{tabular}{|r|r|r|r||r|r||r|r|r|r||r|}
\hline$s_{1}$ & $s_{2}$ & $s_{3}$ & $s_{4}$ & $h_{1}$ & $h_{2}$ & $n$ & $t$ & $\kappa$ & $u$ & $d_{t}(\kappa)$ \\
\hline 1 & 4 & 1 & 5 & 3 & 1 & 8 & 3 & 0 & 17 & 56 \\
2 & 4 & 3 & 3 & 1 & 3 & 15 & 7 & 1 & 30 & 30030 \\
3 & 1 & 1 & 4 & 1 & 3 & 3 & 2 & 0 & 9 & 3 \\
3 & 2 & 1 & 7 & 1 & 3 & 24 & 5 & 0 & 39 & 42504 \\
3 & 3 & 1 & 10 & 1 & 3 & 63 & 8 & 0 & 87 & 3872894697 \\
3 & 3 & 2 & 5 & 1 & 3 & 21 & 8 & 1 & 39 & 813960 \\
3 & 4 & 1 & 13 & 1 & 3 & 120 & 11 & 0 & 153 & 1160681786387760 \\
3 & 4 & 1 & 13 & 3 & 1 & 22 & 11 & 2 & 43 & 17907120 \\
4 & 4 & 1 & 17 & 3 & 1 & 29 & 15 & 3 & 56 & 11417105700 \\
\hline
\end{tabular}

Table 1: Plateaus with small $s_{1}$ and $s_{2}$

As a demonstration of the procedure, Table 1 presents the plateaus and their parameters when $s_{1}, s_{2} \leq 4$.

A class of the plateaus can be explicitly given by $\left(s_{1}, s_{2}, s_{3}, s_{4}\right)=(3 \nu, 1,1,3 \nu+1)$ and $\left(h_{1}, h_{2}\right)=(1,3)$, further by

$$
(n, t, \kappa)=(3 \nu, 3 \nu-1, \nu-1), \quad \nu=1,2, \ldots
$$




\begin{tabular}{|l||r|r|r|r|r|r|r|r|r|r|r|r|r|r|r|r|r|r|r|}
\hline$n$ & 8 & 10 & 15 & 15 & 17 & 21 & 22 & 24 & 24 & 25 & 28 & 29 & 29 & 31 & 34 & 35 & 36 & 36 & 38 \\
\hline$t$ & 3 & 6 & 4 & 7 & 10 & 8 & 11 & 5 & 14 & 19 & 9 & 15 & 20 & 18 & 13 & 6 & 10 & 19 & 22 \\
\hline$\kappa$ & 0 & 1 & 0 & 1 & 2 & 1 & 2 & 0 & 3 & 5 & 1 & 3 & 5 & 4 & 2 & 0 & 1 & 4 & 5 \\
\hline
\end{tabular}

Table 2: Plateaus with $n \leq 40$ appearing no in (9)

The method described above provides the triples $(n, t, \kappa)$ in no ascendent order of layers $n$. We collected here, in Table 2 the levels $n \leq 40$ containing plateaus given no by (9). (Here $s_{1}, s_{2} \leq 23$.)

Note, that the levels

$$
n=0,1,2,4,5,7,11,13,14,16,19,20,23,26,32,37,40, \ldots
$$

of the pyramid do not contain plateaus of diagonal sequences.

It is worth noting, that level $n=15$ is the first layer with 3 diagonal plateaus (see Figure 3$) ; n=66$ is the first with $5 ; n=99$ is the first with $7 ; n=372$ is the first with 11 diagonal plateaus.

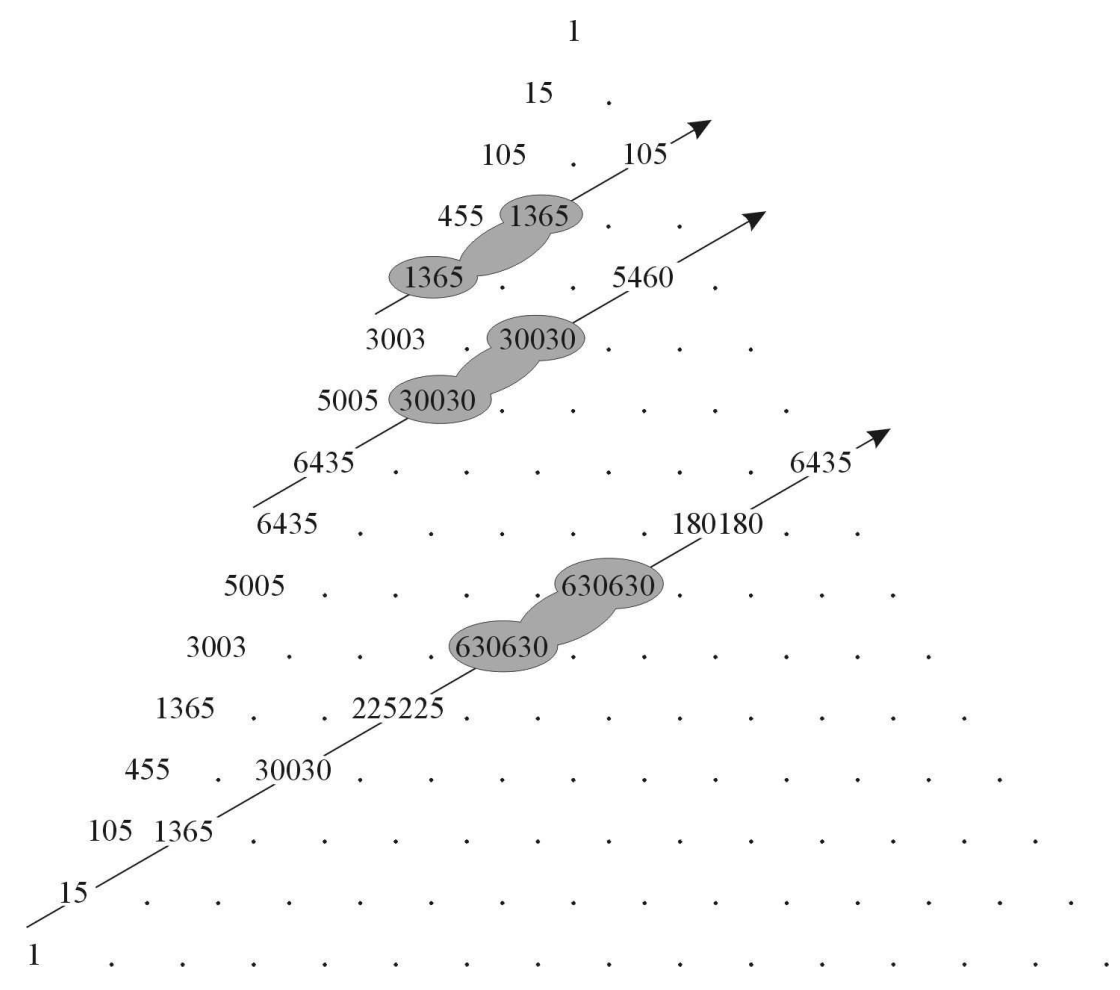

Figure 3: $15^{\text {th }}$ level of regular Pascal pyramid

Acknowledgement We would like to thank the anonymous referee for his/her helpful comments. 


\section{References}

[1] H. Belbachir, H. Bencherif and L. Szalay, Unimodality of certain sequences connected to binomial coefficients, J. Integer Seq., 10 (2007), Article 07.2.3.

[2] H. Belbachir and L. Szalay, Unimodal rays in the ordinary and generalized Pascal triangles, J. Integer Seq., 11 (2008), Article 08.2.4.

[3] M. Benoumhani, A sequence of binomial coefficients related to Lucas and Fibonacci numbers, J. Integer Seq., 6 (2003), Article 03.2.1.

[4] F. Brenti, Unimodal, log-concave, and Pólya frequency sequences in combinatorics, Mem. Amer. Math. Soc. 81 (1989), 413.

[5] S. Tanny and M. Zuker, On a unimodality sequence of binomial coefficients, Discrete Math., 9 (1974), 79-89.

[6] Y. Wang and Y. Yeh, Log-concavity and LC-positivity, J. Comb. Theory, A 114 (2007), 195-210. 